\title{
Loci and genes involved in chronic musculoskeletal pain identified via analysis of genetically independent pain phenotypes
}

\author{
Yakov A. Tsepilov \\ Laboratory of Theoretical and Applied \\ Functional Genomics \\ Novosibirsk State University \\ Novosibirsk, Russia \\ tsepilov@bionet.nsc.ru \\ Sodbo Z. Sharapov \\ Laboratory of Theoretical and Applied \\ Functional Genomics \\ Novosibirsk State University \\ Novosibirsk, Russia \\ Lennart C. Karssen \\ PolyOmica \\ 's-Hertogenbosch, the Netherlands \\ Yurii S. Aulchenko \\ Laboratory of Recombination and \\ Segregation Analysis \\ Institute of Cytology and Genetics SB \\ RAS \\ Novosibirsk, Russia
}

\author{
Maxim B. Freidin \\ Department of Twin Research and \\ Genetic Epidemiology, School of Life \\ Course Sciences \\ King's College London \\ London, UK \\ Elizaveta E. Elgaeva \\ Laboratory of Theoretical and Applied \\ Functional Genomics \\ Novosibirsk State University \\ Novosibirsk, Russia \\ Pradeep Suri \\ Division of Rehabilitation Care \\ Services \\ VA Puget Sound Health Care System \\ Seattle, USA
}

\author{
Alexandra S. Shadrina \\ Laboratory of Theoretical and Applied \\ Functional Genomics \\ Novosibirsk State University \\ Novosibirsk, Russia \\ Jan van Zundert \\ Department of Anesthesiology and Pain \\ Medicine \\ Maastricht University Medical Centre \\ Maastricht, The Netherlands \\ Frances M.K. Williams \\ Department of Twin Research and \\ Genetic Epidemiology, School of Life \\ Course Sciences \\ King's College London \\ London, UK
}

\begin{abstract}
We have evaluated four genetically independent pain phenotypes of four common chronic musculoskeletal pains (GIPs). We assume that the first GIP represents a biopsychological component of chronic musculoskeletal pain, related to physiological and psychological aspects and possibly reflecting pain perception and processing.
\end{abstract}

Keywords - musculoskeletal pains; genome-wide association study; UK Biobank; pleiotropy

\section{Introduction}

Pain is an unpleasant sensory experience caused by tissue damage (or by the threat of its occurrence). It is an adaptive response of organism to the pathogenic influence, which activates various protection mechanisms. Pain and especially chronic pain, conventionally defined as a pain that lasts for 3 and more months sequentially, has a negative impact on all spheres of human life. However, nowadays little is known about the mechanisms of chronic pain onset. Somewhat, it is caused by the fact, that genetic studies of pain are rather complicated due to the high complexity and heterogeneity of the phenotype. Nevertheless, recent studies have shown, that chronic pain is a complex genetic trait with heritability up to $60 \%$, based on different estimates. Moreover, it was found, that various types of chronic pain have a number of genetic factors in common. In this research we decided to apply the method of principal component analysis (PCA) to reduce the heterogeneity and studying of commonalities and pathways that are shared by distinct, although related, pain phenotypes.

The aim of this work was to study the main genetic component that underlies the risk of chronic musculoskeletal pain observed at four different sites: back, neck/shoulder, hip, and knee, using data from the UK Biobank.

\section{Materials and methods}

This work is based on the results of genome-wide association studies in the UK Biobank [1] data with total sample size of 434 thousand people, split into a discovery set of 256,000 people and a replication set of 178,000 people. The matrix of genetic correlations for four chronic musculoskeletal pains was calculated using LD Score regression [2], then the principal components analysis (PCA) was applied to compute the genetically independent pain phenotypes (GIPs). The meta-analysis was conducted using METAL [3]. The further in silico functional annotation of GIPs was performed using DEPICT [4], COJO [5] and GWAS-MAP platform [6].

\section{Results}

We have evaluated four GIPs of four chronic musculoskeletal pains. Six loci were shown to be associated with the first genetic principal component (GIP1), five of them were replicated. Also we have found three loci, associated with the second genetic principal component (GIP2) with one of them being replicated. We identified 13 genes located near the lead SNPs $( \pm 250 \mathrm{~kb})$ in found loci. Most of them were shown by us to be related to musculoskeletal disorders, nervous system and skeletal development. Results of DEPICT analysis for SNP sets associated with GIP1 at P $<5.0 \mathrm{e}-08$ and $\mathrm{P}<1.0 \mathrm{e}-05$ have identified a significant enrichment of terms, related to nervous system and its development and to sensory organ morphogenesis, while the DEPICT analysis of other GIPs did not provide any significant results. The analysis of genetic correlations for GIPs has revealed high correlations between GIP1 and different anthropometric, sociodemographic and psychiatric human traits. The other GIPs 
turned to be genetically correlated with various morphological and anthropometric traits.

We speculate that in the framework of the conventional biopsychosocial model of the chronic pain, the GIP1 relates to the physiological ("bio-") and psychological aspects and is likely reflecting pain perception and pain processing.

\section{ACKNOWLEDGMENT}

The work of SZS was supported by the Russian Ministry of Education and Science under the 5-100 Excellence Programme. The work of YSA was supported by the Federal Agency of Scientific Organizations via the Institute of Cytology and Genetics (project 0324-2019-0040-C-01 / AAAA-A17-117092070032-4). The work of YAT, ASSh, and EEE was supported by the Russian Foundation for Basic Research (project 19-015-00151). The contribution of LCK was funded by PolyOmica. Dr. Suri was supported by VA Career Development Award \# 1IK2RX001515 from the United States (U.S.) Department of Veterans Affairs Rehabilitation Research and Development (RR\&D) Service. Dr. Suri is a Staff Physician at the VA Puget Sound Health Care System. The contents of this work do not represent the views of the U.S. Department of Veterans Affairs or the United States Government.

\section{REFERENCES}

[1] C. Sudlow et al. (2015) UK Biobank: An Open Access Resource for Identifying the Causes of a Wide Range of Complex Diseases of Middle and Old Age, PLOS Medicine, 12: e1001779.

[2] B. Bulik-Sullivan et al. (2015) An atlas of genetic correlations across human diseases and traits, Nature Genetics, 47: 1236-1241.

[3] C.J. Willer et al. (2010) METAL: fast and efficient meta-analysis of genomewide association scans, Bioinformatics, 26: 2190-2191.

[4] T.H. Pers et al. (2015) Biological interpretation of genome-wide association studies using predicted gene functions, Nature Communications., 6: 5890.

[5] J. Yang et al. (2013) Conditional and joint multiple-SNP analysis of GWAS summary statistics identifies additional variants influencing complex traits Genetic Investigation of ANthropometric Traits (GIANT) Consortium 4, DIAbetes Genetics Replication And Metaanalysis (DIAGRAM), Nature Genetics, 44: 369-372.

[6] Gorev DD, Shashkova TI, Pakhomov E, Torgasheva A, Klaric L, Severinov A, et al. GWAS-MAP: a platform for storage and analysis of the results of thousands of genome-wide association scans. Bioinformatics of Genome Regulation and Structure/Systems Biology (BGRS/SB-2018) The Eleventh International Conference. Novosibirsk: ICG SB RAS; 2018. p. 43. doi:10.18699/BGRSSB-2018020 